\title{
DESEMPENHO DE ESCOLARES COM DISLEXIA DO DESENVOLVIMENTO EM TAREFAS FONOLÓGICAS E SILÁBICAS
}

\author{
Performance of students with developmental dyslexia \\ in phonological and syllabic tasks
}

\author{
Giseli Donadon Germano (1), Fábio Henrique Pinheiro ${ }^{(2)}$, Simone Aparecida Capellini ${ }^{(3)}$
}

\begin{abstract}
RESUMO
Objetivo: caracterizar o desempenho em tarefas fonológicas e silábicas de escolares com dislexia do desenvolvimento e comparar estes achados com o desempenho de discentes com bom desempenho escolar. Métodos: participaram do estudo 26 alunos de oito a 12 anos de idade, de ambos os sexos, de 2ª ${ }^{a}$. a ${ }^{a}$. séries do Ensino Fundamental municipal na cidade de Marilia-SP, divididas em GI: composto por 13 escolares com dislexia atendidos no Centro de Estudos da Educação e Saúde - CEES/ UNESP e Gll: composto por 13 alunos com bom desempenho acadêmico, pareados segundo sexo, idade e escolaridade com o GI. Como procedimento foi utilizada a Prova de Consciência Fonológica - Instrumento de Avaliação Seqüencial - CONFIAS. Os resultados foram analisados estatisticamente pelo Teste Mann-Whitney (comparação entre os grupos) e Teste dos Postos Sinalizados-Wilcoxon (comparação entre as variáveis). Resultados: os resultados evidenciaram diferença estatisticamente significante, sugerindo melhor desempenho do Gll em relação ao Gl quanto às tarefas fonêmicas e silábicas. O Gl apresentou diferença estatisticamente significativa nas tarefas silábicas e fonêmicas, com melhor desempenho nas primeiras. Entre os escolares do Gll não houve grande diferença estatística entre tarefas silábicas, apenas entre tarefas fonêmicas. Conclusão: o estudo concluiu que escolares com dislexia do desenvolvimento apresentam dificuldades quanto à identificação de rima e produção de palavras com o som dado, apontando para um déficit em acessar os códigos e as representações fonológicas.
\end{abstract}

DESCRITORES: Aprendizagem; Dislexia; Educação

\section{INTRODUÇÃO}

Aprender a ler e a escrever em uma língua alfabética significa que a criança deve entender que as

(1) Fonoaudióloga; Bolsista Coordenação de Aperfeiçoamento de Pessoal de Nível Superior; Mestranda em Educação, Programa de Pós-Graduação em Educação da Faculdade de Filosofia e Ciências da Universidade Estadual Paulista, UNESP, Marília, SP.

(2) Fonoaudiólogo; Mestrando em Educação, Programa de Pós-Graduação em Educação da Faculdade de Filosofia e Ciências da Universidade Estadual Paulista, UNESP, Marília, SP.

(3) Fonoaudióloga; Docente do Departamento de Fonoaudiologia e do Programa de Pós-Graduação em Educação da Faculdade de Filosofia e Ciências da Universidade Estadual Paulista, UNESP, Marília, SP; Doutora e Pós-doutoranda em Ciências Médicas pela Universidade Estadual de Campinas.

Conflito de Interesses: INEXISTENTE pequenas unidades de fala, denominados fonema, correspondem a letras. A consciência de que a língua é composta de pequenos sons é fundamental para aprender a ler e produzir a escrita alfabética. A proficiência em decodificação de palavras em segmentos menores tem relação com melhor desempenho futuro em leitura ${ }^{1-4}$.

O sistema alfabético de escrita associa um componente auditivo fonêmico a um componente visual gráfico (correspondência grafofonêmica) e para a compreensão do princípio alfabético são necessários três fatores: a consciência de que é possível segmentar a língua falada em unidades distintas; o conhecimento de que essas mesmas unidades repetem-se em diferentes palavras faladas e a ciência das regras de correspondência entre grafemas e fonemas. Destaca-se que os dois primeiros fatores são aspectos da consciência fonológica, e isto a 
coloca como indispensável no desenvolvimento da leitura e da escrita ${ }^{5,6}$

A World Federation of Neurology, em 1968, definiu dislexia como sendo "transtorno de aprendizagem da leitura que ocorre, apesar de inteligência normal, de ausência de problemas sensoriais ou neurológicos, de instrução escolar adequada, de oportunidades sócio-culturais suficientes, além disso, depende da existência de perturbação de aptidões cognitivas fundamentais, frequentemente de origem constitucional" 7 .

A habilidade fonológica vem sendo discutida nas últimas décadas como sendo uma habilidade importante para a aquisição da leitura. A maioria dos indivíduos com atraso em leitura e dislexia apresenta alterações nessa habilidade. A hipótese do déficit fonológico tem sido sustentada por inúmeros trabalhos que têm identificado atrasos quanto à sensibilidade à rima, aliteração e segmentação fonêmica durante o desenvolvimento da leitura ${ }^{8-12}$. No Brasil, os estudos com dislexia têm apontado atrasos quanto à rima, aliteração, manipulação e segmentação fonêmica ${ }^{13-16}$.

Apesar de a literatura enfocar a hipótese do déficit fonológico para o entendimento das manifestações da dislexia, não há consenso sobre a origem deste déficit, ou seja, se o mesmo é decorrente da hipótese do déficit no processamento auditivo 17-19 ou se o mesmo é decorrente da hipótese do déficit específico para percepção da fala ${ }^{20,21}$. A diferença entre as duas hipóteses está no fato de que, na primeira, a criança com dislexia apresenta alterada a discriminação dos sons e, por isso, a fusão rápida de estímulos encontra-se comprometida, resultando em dificuldade para organizar a sequência dos sons na formação das palavras (processamento temporal alterado), enquanto que, na segunda hipótese, a dificuldade da criança disléxica está no mecanismo gerativo para formação de palavras a partir de segmentos menores, como fonemas e sílabas. A discriminação auditiva encontra-se preservada.

Apesar desta controvérsia, vários autores apontam que as crianças com dislexia apresentam dificuldades no uso da rota sublexical para a leitura, ou seja, no uso do mecanismo de conversão grafemafonema em atividades que exigem habilidades fonológicas, como em leitura de palavras inventadas ou na categorização de palavras quanto aos sons ou sílabas ${ }^{2,16,22-25}$.

As diferenças individuais em vocabulário, repetição de não-palavras e consciência fonológica são fatores importantes para o prognóstico do desenvolvimento da leitura e das habilidades relacionadas. $O$ que as pesquisas mostram é que o caminho mais efetivo para a aprendizagem da leitura passa pelo desenvolvimento da consciência fonêmica ${ }^{26}$.
Com base no exposto acima, este estudo tem por objetivos caracterizar o desempenho em tarefas fonológicas e silábicas de escolares com dislexia do desenvolvimento e comparar estes achados com o desempenho de alunos com bom desempenho escolar.

\section{MÉTODOS}

Participaram deste estudo 26 escolares na faixa etária de oito a 12 anos, de ambos os sexos, da $2^{\mathrm{a}}$ a $4^{\mathrm{a}}$ séries do Ensino Fundamental municipal da cidade de Marilia-SP (Tabela 1). Os escolares foram divididos em:

- Grupo I (GI): composto por 13 escolares com dislexia do desenvolvimento atendidos no Centro de Estudos da Educação e Saúde - CEES/UNESP.

$O$ diagnóstico de dislexia do desenvolvimento desses indivíduos foi realizado por equipe interdisciplinar do Centro de Estudos da Educação e Saúde - CEES/UNESP - Marília e Ambulatório de Neurologia Infantil - Aprendizagem do Hospital das Clínicas da Faculdade de Medicina da UNESP-Botucatu, incluindo avaliação fonoaudiológica, neurológica, pedagógica, neuropsicológica e de imagem.

- Grupo II (GII): composto por 13 escolares com bom desempenho acadêmico, pareados segundo sexo, idade e escolaridade com o GI. Os professores escolheram aqueles que apresentaram conceito suficiente em pelo menos 2 bimestres consecutivos e que não apresentavam alterações auditiva e visual descritas em prontuário escolar.

Neste estudo foram realizados os seguintes procedimentos:

a) Termo de Consentimento Pós-informado: Conforme resolução do Conselho Nacional de Saúde CNS 196/96, anterior ao início das avaliações, os pais ou responsáveis dos pacientes selecionados assinaram o termo de Consentimento Pós-informado para autorização da realização do estudo.

b) Prova de Consciência Fonológica - Instrumento de Avaliação Sequencial (CONFIAS) ${ }^{27}$. Esta prova tem duas partes, sendo a primeira correspondente à consciência silábica, composta de nove itens: síntese, segmentação, identificação de sílaba inicial, identificação de rima, produção de palavra com a sílaba dada, identificação de sílaba medial, produção de rima, exclusão, transposição. A segunda parte diz respeito ao reconhecimento dos fonemas, disposta em 7 itens: produção de palavra que inicia com o som dado, identificação de fonema inicial, identificação de fonema final, exclusão, síntese, segmentação e transposição.

A pontuação da prova foi realizada em protocolo específico. As respostas corretas valeram um 
Tabela 1 - Distribuição dos grupos segundo gênero, escolaridade e média etária

\begin{tabular}{lcccccc}
\hline \multirow{2}{*}{ Grupos } & \multicolumn{2}{c}{ Sexo } & \multirow{3}{*}{ Média etária } & \multicolumn{3}{c}{ Escolaridade } \\
\cline { 2 - 3 } & Feminino & Masculino & & 2 & 3 & $\mathbf{4}^{\mathbf{a}}$ \\
\hline GI & $30 \%$ & $70 \%$ & 10 anos & $20 \%$ & $60 \%$ & $20 \%$ \\
GII & $30 \%$ & $70 \%$ & 10 anos & $20 \%$ & $60 \%$ & $20 \%$ \\
\hline
\end{tabular}

Legenda: (GI): escolares com dislexia do desenvolvimento, (GII): escolares com bom desempenho acadêmico

ponto e as incorretas não valeram nada. Na parte da sílaba, o máximo de pontuação foi de 40 e, na parte do fonema, 30 , totalizando 70 pontos, correspondente a $100 \%$ de acertos.

O procedimento foi aplicado foi realizado no Centro de Estudos da Educação e da Saúde - CEES/ Unesp - Marilia, pelos próprios pesquisadores em horário contrário ao da escola. Foi realizada uma sessão para aplicação do procedimento, com duração média de 60 minutos, durante o mês de novembro do ano de 2006.

Este estudo foi realizado após aprovação do Comitê de Ética em Pesquisa da Faculdade de Filosofia e Ciências da Universidade Estadual Paulista - CEP/FFC/UNESP sob o protocolo número 3118/2006.

A análise estatística dos resultados foi realizada por meio da aplicação do Teste Mann-Whitney, com o intuito de verificar possíveis diferenças entre as médias dos grupos, como também o Teste dos Postos Sinalizados-Wilcoxon, com a finalidade de comparar o desempenho dos grupos inseridos nas variáveis de interesse.

Neste estudo foi adotado o nível de significância de $5 \%(0,05)$, para a aplicação dos testes estatísticos, ou seja, quando o valor da significância calculada $(p)$ for menor do que $5 \%(0,05)$, foi considerada uma diferença dita 'estatisticamente significante' marcada com asterisco; e quando o valor da significância calculada (p) for igual ou maior do que $5 \%(0,05)$ foi considerada uma diferença dita "estatisticamente não-significante". Os resultados estatisticamente significantes serão assinalados com asterisco (*).

Para a realização da análise estatística foi utilizado o programa SPSS (Statistical Package for Social Sciences), em sua versão 13.0, a fim de obter os resultados estatísticos.

\section{RESULTADOS}

A Tabela 2 apresenta o desempenho dos escolares do Gl e Gll nas tarefas fonológicas e silábicas do CONFIAS ${ }^{27}$.

Com relação às tarefas silábicas, foi possível verificar que, com a aplicação do Teste Mann-Whitney, não houve diferença estatisticamente significante nas tarefas de síntese, segmentação, identificação, rima e exclusão comparando-se o desempenho do Gl em relação ao Gll. Entretanto, ocorreu diferença estatisticamente significante na tarefa de transposição silábica, apontando para melhor desempenho dos escolares do Gll em relação ao Gl.

Nas tarefas fonêmicas, foi possível verificar que não houve diferença estatisticamente significante nos subtestes de produção e identificação (fonema inicial e final), apontando desempenho semelhante entre os dois grupos. Contudo, observou-se diferença estatisticamente significante entre o Gl e o Gll em relação às provas de exclusão, síntese, segmentação e transposição, tendo ocorrido melhor desempenho dos escolares do Gll em relação ao GI.

A Tabela 3 apresenta a comparação entre as tarefas silábicas e fonêmicas correpondentes dos escolares do Gl.

Com a aplicação do Teste dos Postos Sinalizados de Wilcoxon, foi possível verificar que houve diferença estatisticamente significante quando comparada as tarefas silábicas e fonêmicas correspondentes, o que indica que os escolares do Gl apresentaram melhor desempenho em identificação de rima do que produção de rima, produção de palavras com a sílaba dada do que com o som dado, exclusão silábica do que exclusão fonêmica, síntese fonêmica do que segmentação fonêmica, transposição silábica do que transposição fonêmica.

A Tabela 4 apresenta a comparação entre as tarefas silábicas e fonêmicas correpondentes aos escolares do Gll.

Com a aplicação do Teste dos Postos Sinalizados de Wilcoxon, foi possível verificar que houve diferença estatisticamente significante quando comparada as tarefas silábicas e fonêmicas correspondentes, mostrando que os escolares do GII apresentaram melhor desempenho em exclusão silábica do que exclusão fonêmica, síntese fonêmica do que segmentação fonêmica, transposição silábica do que transposição fonêmica.

\section{DISCUSSÃO}

A dislexia do desenvolvimento é uma dificuldade específica de leitura de causa genética e 
Tabela 2 - Distribuição da média, desvio-padrão e valor de p do desempenho dos escolares dos Gl e GII nas tarefas fonológicas e silábicas do CONFIAS

\begin{tabular}{|c|c|c|c|c|}
\hline Variável & Grupo & Média & Desvio-padrão & Valor de $p$ \\
\hline \multirow{2}{*}{ S1 } & I & 3,62 & 1,12 & \multirow{2}{*}{0,518} \\
\hline & II & 3,92 & 0,28 & \\
\hline \multirow{2}{*}{$\mathbf{S 2}$} & I & 4,00 & 0,00 & \multirow{2}{*}{$>0,999$} \\
\hline & II & 4,00 & 0,00 & \\
\hline \multirow{2}{*}{ S3 } & I & 3,15 & 1,14 & \multirow{2}{*}{0,778} \\
\hline & II & 3,38 & 0,77 & \\
\hline \multirow{2}{*}{ S4 } & I & 3,08 & 1,12 & \multirow{2}{*}{0,656} \\
\hline & II & 3,23 & 1,17 & \\
\hline \multirow{2}{*}{ S5 } & I & 3,77 & 0,60 & \multirow{2}{*}{0,708} \\
\hline & II & 3,77 & 0,44 & \\
\hline \multirow{2}{*}{ S6 } & I & 2,69 & 1,18 & \multirow{2}{*}{0,085} \\
\hline & II & 3,46 & 0,66 & \\
\hline \multirow{2}{*}{ S7 } & $\mathbf{I}$ & 1,85 & 1,21 & \multirow{2}{*}{0,331} \\
\hline & II & 2,38 & 1,39 & \\
\hline \multirow{2}{*}{ S8 } & I & 4,77 & 3,44 & \multirow{2}{*}{0,132} \\
\hline & II & 6,77 & 2,24 & \\
\hline \multirow{2}{*}{ S9 } & I & 2,08 & 1,85 & \multirow{2}{*}{$0,029^{*}$} \\
\hline & II & 3,54 & 1,13 & \\
\hline \multirow{2}{*}{ F1 } & I & 2,69 & 1,44 & \multirow{2}{*}{0,128} \\
\hline & II & 3,46 & 0,88 & \\
\hline \multirow{2}{*}{ F2 } & $\mathbf{I}$ & 2,85 & 1,28 & \multirow{2}{*}{0,101} \\
\hline & II & 3,62 & 0,65 & \\
\hline \multirow{2}{*}{ F3 } & I & 2,23 & 1,30 & \multirow{2}{*}{0,063} \\
\hline & II & 3,08 & 1,32 & \\
\hline \multirow{2}{*}{ F4 } & I & 1,54 & 2,11 & \multirow{2}{*}{$0,011^{*}$} \\
\hline & II & 3,69 & 2,14 & \\
\hline \multirow{2}{*}{ F5 } & I & 1,31 & 1,25 & \multirow{2}{*}{$0,004^{*}$} \\
\hline & II & 2,77 & 0,83 & \\
\hline \multirow{2}{*}{ F6 } & 1 & 0,31 & 0,85 & \multirow{2}{*}{$0,003^{*}$} \\
\hline & II & 1,77 & 1,48 & \\
\hline F7 & $I$ & 0,08 & 0,28 & $0,001^{*}$ \\
\hline & II & 1,85 & 1,46 & \\
\hline
\end{tabular}

Legenda: S1 = síntese silábica, S2= segmentação silábica, S3= identificação de sílaba inicial, S4= identificação de rima, S5= produção de palavras com sílaba dada, S6= identificação de silaba medial, S7= produção de rima, S8= exclusão de sílaba, S9= transposição de sílabas, $\mathrm{F} 1=$ produção de palavras com som dado, F2= identificação de fonema inicial, F3=identificação de fonema inicial, F4= exclusão de fonema, F5= síntese fonêmica, F6= segmentação fonêmica, F7= transposição fonêmica. Análise estatística: Teste Mann-Whitney.

neurológica que compromete principalmente 0 desenvolvimento da leitura. As habilidades necessárias para o desenvolvimento da leitura em um sistema de escrita alfabético, como o português brasileiro, incluem o acesso ao léxico mental, à memória de trabalho e à consciência fonológica que, quando se encontram alteradas, comprometem diretamente o domínio do sistema de escrita em nível fonológico e ortográfico ${ }^{5,6,28-30}$.

Os resultados deste estudo revelaram que as crianças com dislexia do desenvolvimento apresen- taram dificuldades semelhantes ao grupo de escolares com bom desempenho acadêmico no que se refere à exclusão, síntese e transposição fonêmica, sugerindo que tais dificuldades metalinguísticas não podem ser consideradas características de escolares com quadro de dislexia do desenvolvimento, pois, quando o princípio alfabético não é inserido no contexto da alfabetização como instrução formal, podem ocorrer dificuldades na percepção fonológica necessária para a aprendizagem da leitura em um sistema de escrita alfabético como o 
Tabela 3 - Distribuição da média, desvio-padrão e valor de p referente à comparação entre as tarefas silábicas e correspondentes dos escolares do GI

\begin{tabular}{|c|c|c|c|}
\hline Par de Variáveis & Média & Desvio-padrão & Valor de $p$ \\
\hline $\mathrm{s} 1$ & 3,62 & 1,12 & \multirow{2}{*}{0,180} \\
\hline S2 & 4,00 & 0,00 & \\
\hline S3 & 3,15 & 1,14 & \multirow{2}{*}{0,132} \\
\hline s6 & 2,69 & 1,18 & \\
\hline S4 & 3,08 & 1,12 & \multirow{2}{*}{$0,015^{\star}$} \\
\hline s7 & 1,85 & 1,21 & \\
\hline S5 & 3,77 & 0,60 & \multirow{2}{*}{$0,016^{*}$} \\
\hline $\mathbf{F} 1$ & 2,69 & 1,44 & \\
\hline F2 & 2,85 & 1,28 & \multirow{2}{*}{0,155} \\
\hline F3 & 2,23 & 1,30 & \\
\hline S8 & 4,77 & 3,44 & \multirow{2}{*}{$0,005^{\star}$} \\
\hline F4 & 1,54 & 2,11 & \\
\hline F5 & 1,31 & 1,25 & \multirow{2}{*}{$0,033^{*}$} \\
\hline F6 & 0,31 & 0,85 & \\
\hline s9 & 2,08 & 1,85 & \multirow{2}{*}{$0,011^{*}$} \\
\hline F7 & 0,08 & 0,28 & \\
\hline
\end{tabular}

Legenda: S1 = síntese silábica, S2= segmentação silábica, S3= identificação de sílaba inicial, S4= identificação de rima, S5= produção de palavras com sílaba dada, S6= identificação de silaba medial, S7= produção de rima, S8= exclusão de sílaba, S9= transposição de sílabas, F1= produção de palavras com som dado, F2= identificação de fonema inicial, F3= identificação de fonema inicial, F4= exclusão de fonema, F5= síntese fonêmica, F6= segmentação fonêmica, F7= transposição fonêmica. Análise estatística: Teste dos Postos Sinalizados de Wilcoxon

Tabela 4 - Distribuição da média, desvio-padrão e valor de p referente à comparação entre as tarefas silábicas e correspondentes dos escolares do Gll

\begin{tabular}{|c|c|c|c|}
\hline Par de Variáveis & Média & Desvio-padrão & Valor de $p$ \\
\hline $\mathrm{s1}$ & 3,92 & 0,28 & \multirow{2}{*}{0,317} \\
\hline S2 & 4,00 & 0,00 & \\
\hline S3 & 3,38 & 0,77 & \multirow{2}{*}{0,739} \\
\hline s6 & 3,46 & 0,66 & \\
\hline S4 & 3,23 & 1,17 & \multirow{2}{*}{0,076} \\
\hline s7 & 2,38 & 1,39 & \\
\hline S5 & 3,77 & 0,44 & \multirow{2}{*}{0,260} \\
\hline $\mathbf{F} 1$ & 3,46 & 0,88 & \\
\hline F2 & 3,62 & 0,65 & \multirow{2}{*}{0,084} \\
\hline F3 & 3,08 & 1,32 & \\
\hline S8 & 6,77 & 2,24 & \multirow{2}{*}{$0,002^{*}$} \\
\hline F4 & 3,69 & 2,14 & \\
\hline F5 & 2,77 & 0,83 & \multirow{2}{*}{$0,028^{*}$} \\
\hline F6 & 1,77 & 1,48 & \\
\hline s9 & 3,54 & 1,13 & \multirow{2}{*}{$0,003^{*}$} \\
\hline F7 & 1,85 & 1,46 & \\
\hline
\end{tabular}

Legenda: S1 = síntese silábica, S2= segmentação silábica, S3= identificação de sílaba inicial, S4= identificação de rima, S5= produção de palavras com sílaba dada, $\mathrm{S} 6=$ identificação de silaba medial, $\mathrm{S} 7=$ produção de rima, $\mathrm{S} 8=$ exclusão de sílaba, $\mathrm{S} 9=$ transposição de sílabas, F1= produção de palavras com som dado, F2= identificação de fonema inicial, F3= identificação de fonema inicial, F4= exclusão de fonema, F5= síntese fonêmica, F6= segmentação fonêmica, F7= transposição fonêmica. Análise estatística: Teste dos Postos Sinalizados de Wilcoxon 
português brasileiro. A dificuldade na descoberta do princípio fonológico (identificação e manipulação de fonemas) ocasiona problemas de leitura no sistema de escrita alfabética ${ }^{8,16,31}$.

Desta forma, o que este estudo evidenciou é que as crianças com dislexia do desenvolvimento apresentaram dificuldades quanto à identificação de rima e produção de palavras com o som dado, apontando para um déficit em acessar os códigos fonológicos, representações fonológicas e de armazenamento fonológico ${ }^{4,28,31-33}$.

O teste de rima envolve a identificação de unidades chamadas intra-sílabas e é realizado a partir da detecção de dois fonemas comuns. Quando essa habilidade encontra-se alterada, é o mecanismo gerativo de produção de palavras a partir de sons e sílabas que se encontra prejudicado ${ }^{31,34}$. Esta dificuldade foi evidenciada nos escolares com dislexia, os quais tiveram problemas em tarefas como identificação de sílabas e fonemas iniciais, mas, principalmente, nas tarefas de identificação das sílabas mediais e fonemas finais, além de identificação de rima e produção de palavras com o som dado, tarefas de exclusão, síntese, segmentação e transposição fonêmica.

Os achados deste estudo vão ao encontro de pesquisas realizadas com escolares disléxicos, tendo evidenciado dificuldades quanto à percepção e produção de rima, segmentação e manipulação fonêmica, mostrando que o déficit fonológico compromete a representação, a análise e a manipulação das informações fonêmicas ${ }^{10-12,15,16}$, que são necessárias para gerar representação interna da estrutura fonológica da palavra a partir da palavra escrita antes mesmo de o significado da palavra ser acessado ${ }^{34}$.

Os achados deste estudo evidenciaram que a verificação de habilidades fonológicas e silábicas de crianças com dislexia do desenvolvimento precisa ser mais estudada na realidade brasileira, pois, somente dessa forma, poderemos fonoaudiologicamente identificar com segurança o perfil dessas crianças quanto às habilidades metalinguísticas, bem como diferenciá-las de crianças que apresentam simples atrasos de leitura.

\section{CONCLUSÃO}

Os resultados deste estudo nos permitiram concluir que as crianças com dislexia do desenvolvimento apresentaram dificuldades semelhantes ao grupo de escolares com bom desempenho acadêmico no que se refere à exclusão, síntese e transposição fonêmica, sugerindo que tais dificuldades metalinguísticas não podem ser consideradas características de discentes com esse quadro, pois, quando o princípio alfabético não é inserido no contexto da alfabetização como instrução formal, podem ocorrer dificuldades na percepção fonológica necessária para a aprendizagem da leitura em um sistema de escrita alfabético como o português brasileiro, como ocorreu entre as crianças com bom desempenho escolar.

As crianças com dislexia do desenvolvimento deste estudo apresentaram dificuldades quanto à identificação de rima e produção de palavras com o som dado, apontando para um déficit em acessar os códigos e as representações fonológicas.

\begin{abstract}
Purpose: to characterize and to compare the performance in phonological and syllabic tasks of developmental dyslexia to good readers. Methods: twenty-six students participated, with age varying between 8 and 12 years, both genders, of $2^{\text {nd }}$ and $4^{\text {th }}$ grades of the municipal elementary education of the city of Marilia-SP. They were divided into Gl: 13 developmental dyslexic students Center of Studies of the Education and Health - CEES/UNESP, and Gll: 13 good readers, paired by gender, age and school level. As procedure we used the Test of Phonological Awareness - Instrument of Sequential Evaluation - CONFIAS. The results were statistically analyzed by Mann-Whitney Test and Wilcoxon Test. Results: the results evidenced significant statistical difference, suggesting better performance of the Gll in relation to the GI in the phonemic and syllabic tasks. The Gl presented statistical significant difference in the syllabics and phonemic tasks, with better performance in the syllabic tasks. The students of the Gll did not have significant statistical difference as for the syllabic tasks, just among phonemic tasks. Conclusions: dyslexic students had difficulties in tasks of identification of rhymes and production of words with given sound, indicating a deficit in the access of phonological codes and phonological representations.
\end{abstract}

KEYWORDS: Learning; Dyslexia; Education 


\section{REFERÊNCIAS}

1. Baddeley A. Working memory and language: an overview. J Commun Disord. 2003; 36(3):189-208.

2. Bradley L, Bryant PE. Categorizing sounds and learning to read: a causal connection. Nature. 1983; 301:419-21.

3. Byrne B, Fielding-Barnsley R. Evaluation of a program to teach phonemic awareness to young children: a 2- and 3-year follow-up and a new preschool trial. J Educ Psychol. 1995; 87(3):488-503.

4. Ortiz R, Jimenez JE, Miranda EG, Rosquete RG, Hernandez-Valle I, Rodrigo $M$, et al. Locus and nature of perceptual phonological deficit in Spanish children with reading disabilities. J Learn Disab. 2007; 40(1):80-92.

5. BarreraSD, MalufMR. Consciênciametalinguística e alfabetização: um estudo com crianças da primeira série do ensino fundamental. Psicol Reflex Crít. 2003; 16(3):491-502.

6. Guimarães SRK. Dificuldades no desenvolvimento da lectoescrita: o papel das habilidades metalinguísticas. Psicol Teor Pesq. 2003 jan/abr; 19(1):33-45.

7. Critchley M. Specific developmental dyslexia. In: Frederiks JAM. Handbook of neurology. Amsterdam: Elsevier; 1985. p.353-64.

8. Thomson JM, Goswami U. Rhythmic processing in children with developmental dyslexia: auditory and motor rhythms link to reading and spelling. $J$ Physiol Paris. 2008. 102(1-3):120-9.

9. Van Leeuwen $T$, Been $P$, Van Herten $M$, Zwarts $F$, Maassen B, Van Der Leij A. Two-month-old infants at risk for dyslexia do not discriminate /bAk/ from /dAk/: a brain-mapping study. J Neurolinguist. 2008; 21(4):333-48.

10. Vukovic RK, Wilson AM, Nash KK. Naming speed deficits in adults with reading disabilities: a test of the double-deficit hypothesis. J Learn Disabil. 2004; 37(5):440-50.

11. Savage RS, Frederickson N, Goodwin R, Patni U, Smith N, Tuersley L Relationships among rapid digit naming, phonological processing, motor automaticity, and speech perception in poor, average, and good readers and spellers. $J$ Learn Disabil. 2005; 38(1):12-28.

12. Swanson HL, Howard CB, Sáez L. Do different components of working memory underlie different subgroups of reading disabilities? J Learn Disabil. 2006; 39(3):252-69.

13. Capellini SA, Ciasca SM. Eficácia do programa de treinamento com a consciência fonológica em crianças com distúrbio específico de leitura e escrita e distúrbio de aprendizagem. Temas Desenvol. 2000; 48(8):17-23.
14. Germano GD. Eficácia do programa de remediação fonológica Play On em crianças com dislexia do desenvolvimento. [dissertação] Marilia (SP): Universidade Estadual Paulista; 2008.

15. Barros AFF, Capellini SA. Avaliação fonológica, de leitura e escrita em crianças com distúrbio específico de leitura. J Bras Fonoaudiol. 2003; 4(14):11-9.

16. Capellini AS, Padula NAMR, Ciasca SM. Desempenho de escolares com distúrbio específico de leitura em programa de remediação. Pró-Fono. 2004; 16(3):261-74.

17. Tallal P. Auditory temporal perception, phonics, and reading disabilities in children. Brain Lang. 1980 mar; 9(2):182-98.

18. Tallal P, Piercy M. Developmental aphasia: the perception of brief vowels and extended stop consonants. Neuropsychol. 1975 jan; 13(1):69-74.

19. Rey V, De Martino S, Espesser R, Habib M. Temporal processing and phonological impairment in dyslexia: effect of phoneme lengthening on order judgment of two consonants. Brain Lang. 2002 mar; 80(3):576-91.

20. Brady S, Shankweiler D, Mann V. Speech perception and memory coding in relation to reading ability. J Exp Child Psychol. 1983; 35(2):345-67.

21. Studdert-Kennedy M, Mody M. Auditory temporal perception deficits in the reading impaired: a critical review of the evidence. Psych Bull Rev. 1995; 2:508-14.

22. Rosen S. Auditory processing in dyslexia and specific language impairment: is there a deficit? What is its nature? Does it explain anything? J Phonetics. 2003; 31(3):509-27.

23. Snowling MJ, Gallagher A, Frith U. Family risk of dyslexia is continuous: individual differences in the precursors of reading skill. Child Develop. 2003 mar; 74(2):358-73.

24. Speece DL, Ritchey KD. A longitudinal study of the development of oral reading fluency in young children at risk for reading failure. J Learn Disab. 2005; 38(5):387-99.

25. Pekkola J, Laasonen M, Ojanen V, Autti T, Jääskeläinen IP, Kujala $T$, et al. Perception of matching and conflicting audiovisual speech in dyslexic and fluent readers: an fMRI study at 3 Tesla. Neuroimag. 2006; 29(3):797-807.

26. Mann VA, Foy J. Speech development patterns and phonological awareness in preschool children. Ann Dyslexia. 2007; 57(1):51-74.

27. Moojen S, Lamprecht R, Santos RM, Freitas GM, Brodacz R, Siqueira M, Costa AC, Guarda E. Consciência fonológica: instrumento de avaliação sequencial. São Paulo: Casa do Psicólogo; 2003. 
28. Capellini SA, Padula NAMR, Santos LCA, Loureceti MD, Ribeiro LA. Familial dyslexia: characterization of phonological, working memory, reading and writing findings. In: Letterman $\mathrm{J}$, organizador. Dyslexia in children: new developments. New York: New Science Publisher; 2006. p. 89-110.

29. Cardoso-Martins C, Pennington BF. The relationship between phoneme awareness and rapid naming skills and literacy acquisition: the role of development period and reading ability. Scient Stud Read. 2004; 8(1):27-52.

30. Gray A, McCutchen D. Young readers' use of phonological information: phonological awareness, memory and comprehension. J Learn Disabil. 2006; 39(4): 325-33.
31. McCrory EJ, Mechelli A, Frith U, Price CJ. More than words: a common neural basis for reading and naming deficits in developmental dyslexia? Brain. 2005; 128(Pt2):261-7.

32. Duncan LG, Seymour PH, Bolik F. Rimes and superrimes: an exploration of children's disyllabic rhyming skills. Br J Psychol. 2007 may; 98(Pt2):199-221.

33. Bryant PE, MacLean M, Bradley LL, Crossland J. Rhyme and alliteration, phoneme detection, and learning to read. Develop Psychol. 1990; 26(3):429-38.

34. Katzir T, Misra M, Poldrack RA. Imaging phonology without print: assessing the neural correlates of phonemic awareness using fMRI. Neuroimag. 2005 aug; 27(1):106-15.

DOI: 10.1590 / S1516-18462008005000004

RECEBIDO EM: 27/07/2007

ACEITO EM: 07/04/2008

Endereço para correspondência:

Giseli Donadon Germano

Rua Frei Jacinto, 264

Marília - SP

CEP: $17501-240$

E-mail: giseliger@yahoo.com.br 